\title{
Study of the Constrained Resources to Projects Based on the Random Networks
}

\author{
Mingyu Li ${ }^{1, a}$, Li Yang ${ }^{2, b}$ \\ ${ }^{1}$ Tianjin College,University of Science and Technology Beijing Tianjin, China \\ ${ }^{2}$ Tianjin College,University of Science and Technology Beijing Tianjin, China
}

\begin{abstract}
In this paper, a number of concurrent network projects with random activities are studied, and the proposed model enables people to control various resources such as rare and expensive resources transported from outside in a short time. For each project, the schedule date and the admissible confidence probability of timely completion of the project were defined. The initial instants of realization of all projects, the required total power of each of the reproducible resources, the delivery schedule of rare reproducible resources that are not at the disposal of the project management system, and the schedule of beginning all activities were determined. The minimal nonoperational costs, such as penalty provisions for failure to execute the project in time, fines for idling of the rare external resources, costs of resource lease were used as the target function. The developed model can be used to plan and supervise one or more random PERT-like network projects. It can significantly reduce the cost of project implementation.
\end{abstract}

\section{Introduction}

There are numerous papers on optimization of allocation and delivery of the resources for network projects with deterministic operations. Recently, there exist some resource models for projects with random durations of operations. These models are more sophisticated mostly compared with the deterministic models because of the difficulties involved in constructing the schedule of resource delivery and consumption. At the same time, the majorities of projects are carried out in the random environment and constrained resources. Therefore, development of more generalized resource models for network projects seems quite topical both from the scientific and applied standpoints.

\section{Problem Descriptions}

The resources involved into the network projects can be classified with two groups [1]:

Expensive and rare resources are Class A resources. The A-resources must be strictly controlled in the sense that their delivery must be scheduled in advance. The Aresource delivery graph must be deterministic, because one cannot envisage even approximately when any activity will be ready for execution.

Reproducible resources are class B-resources. Bresources can be used in one or more project activities at any time $t$, if available. Therefore, the instants of delivering the B-resources to the project activities are random and defined in the course of project execution.

In the paper, the models of planning and supervising the network projects [2] not only include the project schedule dates but also include supervision by probability, that is, the model parameters must include the lower bound on the confidence probability of executing each project by the schedule date. The instants of beginning each project also are among the optimized parameters. This is necessary because the lease of the reproducible Bresources is related with the initial instant of supplying them to the project store. The leased resources are stored from the initial instant (deterministic variable to be optimized) and to the instant of project completion (random variable).

The model developed below includes numerous cost parameters. The operational costs such as the cost of raw materials, electric power, the A-resources and B-resources in the course of project execution, and so on, which are independent of the model of planning or supervision, were not included in the model intentionally. The target function of the model includes the following nonoperational costs.

Costs of leasing and maintaining the B-resources during the time of project realization.

Costs of project storage for each time unit of storage.

Fines on the project management system for idling of the A-resources.

Fine paid once to the customer for failure to execute the project in time.

Fine paid for each project for each unit of the outstanding project time.

\section{Problem Formalization}

Before starting the projects, we produce a $e$-th $(e$ is the number of network projects) random PERT network 
project $G_{e}(N, A)$; the deterministic optimal values of $S_{e}$ (the instant of starting the $e$ th project), $R_{k e}$ (the total power of the $k$ th resource leased for the eth project) and $T\left(i_{\xi A}, j_{\xi A}\right)_{e}$ (the predefined instant of delivering the $A$ resources for the $\left(i_{\xi A}, j_{\xi A}\right)_{e}$ th activity), $1 \leq e \leq v, 1 \leq k \leq m, 1$ $\leq \xi \leq n_{A}$.

In the course of its realization, the random values $S_{i j e}$ (the instant of starting the $(i, j)_{e}$ th activity) for all activities $(i, j)_{e}$, to optimize the target function

$$
\min _{\left\{S_{e}\right\},\left\{R_{k e}\right\}, T\left\{i \xi A, j_{\xi A}\right\}} \bar{C}
$$

$$
\begin{aligned}
& \text { under the constraints } \\
& \operatorname{Pr}\left\{F_{e} \leq D_{e}\right\} \geq p_{e}^{*}, 1 \delta \varepsilon \delta \varpi \\
& S_{i_{\xi_{A}}, j_{\xi_{4}} e} \geq T\left(i_{\xi_{A}}, j_{\xi A}\right)_{e}, 1 \delta\left(\delta v_{A}\right. \\
& S_{i j e} \leq T(i) \subset \forall(i, j)_{e} \subset G_{e}(N, A) \\
& t=S_{i i_{\eta B} j_{\eta B} e}, 1 \leq \eta \leq q \leq n_{B e} \Rightarrow \\
& \sum_{\eta=1}^{q} r_{i i_{\eta B} j_{\eta B} e k} \leq R_{k e}(t)
\end{aligned}
$$

$D_{e}$ is the predefined schedule date of executing the $e$ th project, $p_{e}$ is the predefined confidence probability of executing the eth project in time; $r_{i i_{\eta B} j_{\eta B} e k}$ is the predefined power of the $k$-type $B$-resources consumed by the $\left(i_{\eta B}, j_{\eta B}\right)$ eth activity.

The random variable $C$ satisfies the relationship

$$
\begin{aligned}
& \quad\left[\sum_{\left(i_{\xi A}, j_{\xi A}\right)}\left\{e\left(i_{\xi A}, j_{\xi A}\right)_{e}\left[S_{i_{\xi A} j_{\xi A}}-T\left(i_{\xi A}, j_{\xi A}\right)_{e}\right]\right\}+\right. \\
& C=\sum_{e=1}^{v} \sum_{k=1}^{m}\left[c_{k e} R_{k e}\left(F_{e}-S_{e}\right)\right]+ \\
& \left.\left[C_{e}^{*}+C_{e}^{* *}\left(F_{e}-D_{e}\right)\right] \delta_{e}\right] \\
& +\left[C_{e}^{* * * *}\left(D_{e}-F_{e}\right)\left(1-\delta_{e}\right)\right]
\end{aligned}
$$

where

$\delta_{e}=\left\{\begin{array}{cc}1 & F_{e}>D_{e} \\ 0 & \text { otherwise }\end{array}\right.$

$T(i)$ stands for the instant of the event $i \in G_{e}(N, A)$. $F_{e}$ is the instant of completing the eth project, notably $F_{e}=\max _{(i, j)_{e}} F_{i j e} \cdot C_{e}{ }^{*}$ is the predefined single fine for failure to fulfill the eth project in time for $F_{e}>D_{e} ; C_{e} * *$ is the predefined fine for a unit time of the overdue eth project, that is, during the period $\left[D_{e}, F_{e}\right] ; C_{e}{ }^{* * *}$ are the predefined costs of storing the $e$ th project in unit time if it is executed before the schedule date for $F_{e}<D_{e}$.

\section{Heuristic Algorithms}

In the heuristic algorithm, different projects are assumed independently of each other, which eliminate the possibility of using a unique central store of resources. Under this assumption, the problem (1)-(7) can be solved independently for each project. The resulting optimal mean values of the nonoperational costs $\bar{C}_{e}$ for each $e$ th project can be summed to obtain the desired optimal value

$C$ for the project system as a whole. Here, the values of $S_{e}, R_{k e}$, and $T\left(i_{\xi A}, j_{\xi A}\right)_{e}$ obtained for individual projects are the solution of the global problem (1)-(7).

Two hierarchical levels, external and internal, are introduced in the model. At the upper level, we solve for one project the following problem (called below $P 1$ ). In order to minimize the nonoperational conditional costs with regard for the confidence probability $p^{*}$, it is required to determine the optimal values of $S,\left\{R_{k}\right\}, 1 \leq k \leq m$, and $T\left(i_{\xi A}, j_{\xi A}\right), 1 \leq \xi \leq n_{A}$ :

$\min _{S, R_{k}, T\left\{i \xi A, j_{\xi \xi\}}\right\}}\left\{\overline{\left.\left[C^{o p t} / S,\left\{R_{k}\right\}, T\left\{i \xi A, j_{\xi A}\right)\right\}\right]+\left\{\gamma\left(\bar{p}-p^{*}\right)\right\} K}\right\}$

under the constraints

$$
\begin{aligned}
& R_{k \min } \leq R_{k} \leq R_{k \max } 1 \delta \kappa \delta \mu \\
& S_{\min } \leq S \leq S_{\max }
\end{aligned}
$$

where

- $\overline{C^{o p t}}$ is established at the lower hierarchical level by means of the simulation model combined at the upper level with the extremum-seeking model;

- $\boldsymbol{p}$ which is determined by simulation is the statistical frequency of the estimate of the probability $\operatorname{Pr}_{r}\{F<D\}$ with a fixed set of values $S$, $R_{k}$, and $T\left(i_{\xi_{A}}, j_{\xi_{A}}\right)$;

$$
\gamma(x)=\left\{\begin{array}{cc}
0 & x \geq 0 \\
1 & \text { otherwise }
\end{array}\right.
$$

- $K$ is a very great value (in the experiment, $K=$ $10^{17}$ )

Therefore, the target function (8) automatically eliminates the possibility of $\bar{P}<p^{*}$, that is, takes into account the constraint (2).

To solve $P 1,1+m+n_{A}$ optimized variables are used. First, $S$ is optimized with regard for (9), the rest of the variables being fixed; then, $R_{1}$, with regard for the new fixed values of $S$; then, $R_{2}$, with new fixed values of $S$ and $R_{1}, \ldots$ and so on until $R_{m}$. In doing so, constraints (10) are taken into account. By fixing $(m+1)$ new values, the coordinate wise optimization embraces new variables $T\left(i_{1 A} ; j_{1 A}\right) ; T\left(i_{2 A} ; j_{2 A}\right), \ldots, T\left(i_{n A}, j_{n A}\right)$ till the end of the first iteration. The second iteration begins again from $S$ and so on until the coordinate wise optimization drives the relative error between two adjacent iterations $\bar{C}^{(w)}$ and $\bar{C}^{(w+1)}$ below the admissible error $\varepsilon>0$.

To obtain a representative statistics for estimation of $\bar{C}(\vec{X})$, determination of the new, $\left(1+m+n_{A}\right)$-dimensional point of search $\vec{X}$ periodically makes use of the simulation model at the lower hierarchical level and carries out quite a few simulation runs. Since the $A$ resources are consumed by only a few project activities and the amount, $m$, of the consumed $B$-resources also is not large, at the upper level the total number of optimizing variables is not large and admits coordinate wise optimization. It is needless to say that if the passage to a new search point $\vec{X}$ results in greater target function $\bar{C}$, then the search is carried out from the point $\vec{X}$ which 
until now corresponded to the minimal value $\bar{C}$.

At the lower hierarchical level, the simulation project model with a built in optimizer, the optimal problem $P 2$, is realized. The current search point $\vec{X}$ in the space of 1 $+m+n_{A}$ variables $S, R_{k}$, and $T\left(i_{\xi_{\xi 4}}, j_{\xi_{4}}\right)$ is the input parameter of the model. The simulation model determines the random instants of beginning all project operations $S_{i j}$ with regard for constraints (3)-(5). As for the optimizer, it allocates the free $B$-resources to the ready project activities which consume these resources. The free resources are allocated at the decision points provided that there are activities queued for the resources at the project store.

Execution of the project must be preceded by solving problem $P 1$ at the upper hierarchical level, that is, determining the optimal set of values of $S, R_{k}$, and $T\left(i_{\xi A}, j_{\xi A}\right)$.

The resulting optimal $\bar{C}^{\text {opt }} / S^{\text {opt }}, R_{k}^{\text {opt }}$, $T^{o p t}\left(i_{\xi A}, j_{\xi 4}\right)$ must support the project managers in making decisions about project feasibility (if the management can carry the burden of no operational costs $\bar{C}$ ) or restructuring it, changing its funding, and so on. If the resulting optimal set of parameters is accepted as the plan, then the real project can be supervised, the $B$ resources leased, and the $A$-resources supplied. Therefore, solution of the problem $P 1$ provides the optimal project schedule. As for estimation of the random realization of $S_{i j}$, they are determined by a single run of the simulation model[4].

\section{Simulation Model Of Consumming The B-Resources}

As was noted above, the optimal values of $S, R_{k}$, and $T\left(i_{\xi_{\xi A}}, j_{\xi A}\right)$ are the inputs of the optimal problem $P 2$ which determines supply of the $B$-resources to the project activities. The random realizations $S_{i_{n} j_{n}}$, where $\left\{i_{\eta B}, j_{\eta B}\right\} \equiv\{i, j\} /\left\{i_{\xi_{4}}, j_{\xi_{4}}\right\}$, are the outputs of $P 2$ which is part and parcel of the simulation model of project realization.

\subsection{Simulation Submodel}

1) Determines the instants $t$ of making decisions about the allocation of the available B-resources to the ready project activities (if the available B-resources can be supplied to at least one activity.

2) Queues the ready activities.

3) Supplies the A-resources to the ready activities in compliance with constraints (3), (4).

4) Supplies the available B-resources to the queued activities if the total need for resource of all queued activities does not exceed the free stored resources.

5) Models durations $t_{i j}$ of all project activities $(i, j)$ upon supplying the A-resources or B-resources for their execution. Therefore, the simulation submodel determines both $S_{i j}$ and $F_{i j}=S_{i j}+t_{i j}$.
6) Returns to the project store the released Bresources upon the completion of the current project activity $\left(i_{\eta B}, j_{\eta B}\right)$.

7) Determines (after simulation of $t_{i j}$ and $F_{i j}$ ) the instants of all project events $i$ to estimate $\mathrm{T}(i)$ in constraint (4) by the formula

$$
T(j)=\max _{i \in D(j)}\left\{S_{i j}+t_{i j}\right\}
$$

where $\mathrm{D}(j)$ is the set of events $i$ that immediately precedes the event.

8) Determines by simulation the auxiliary parameters $\mathrm{p}\left(i_{B} ; j_{B}\right)$ for the problem $\mathrm{P} 2$.

\subsection{Optimal Submodel}

It includes $P 2$ and at the instants $t$ of decision making allocates the available $B$-resources to the queued project activities. If at the instant $t$ more than one activity is queued and the free $B$-resources are limited, then one must conduct 'competition' between the activities to establish which of them must be provided with resources in the first place. The problem $P 2$ is formulated in mathematical terms as follows: let at the instant $t$ there be $q<n_{B}$ queued activities $\left(i_{\eta B}, j_{\eta B}\right), 1 \leq \eta \leq q, q>1$, and the relationship

$$
R_{k}(t)<\sum_{\eta=1}^{q} r_{i_{\eta B}, j_{\eta B}}
$$

be valid at least for one of the subscripts $k$, that is, the $B$-resources of the $k$ th type are insufficient to supply all queued activities[5].

In the case of fixed powers $r_{i_{n B}, j_{\eta B}}, 1 \leq k \leq m$, we suggest [3] to solve the classical problem of integer programming by determining the integer $0-1$ values $\rho_{\eta}, 1 \leq \eta \leq q$, maximizing the target function

$$
\max _{\left\{\rho_{\eta}\right\}}\left\{\sum_{\eta=1}^{q}\left[\rho_{\eta} p\left(i_{\eta B}, j_{\eta B}\right) \mu_{i_{\eta B}, j_{\eta B}}\right]\right\}
$$

under the constraints

$$
\sum_{\eta=1}^{q}\left[\rho_{\eta} r_{i_{\eta B} j_{\eta B} k}\right] \leq R_{k}(t) \quad 1 \delta \kappa \delta \mu
$$

where $p\left(i_{\eta B}, j_{\eta B}\right)$ denotes the probability that the activity $\left(i_{\eta B}, j_{\eta B}\right)$ is on the critical path in the course of single realization of the project, that is, realization of one simulation run, and

$$
\rho_{\eta}=\left\{\begin{array}{cc}
1 & \text { the activity }\left(i_{\eta B}, j_{\eta B}\right) \text { is supplied } \\
0 & \text { by the } B \text { - resources } \\
\text { otherwise }
\end{array}\right.
$$

The product $W_{\eta}=\rho\left(i_{\eta B}, j_{\eta B}\right) \mu_{i_{\eta B} j_{\eta B}} \quad$ is the contribution of the activity $\left(i_{\eta B}, j_{\eta B}\right)$ of the mean length value of the critical path. The idea of P2 lies in using model (14)-(16) to choose a subset of queued activities which minimizes the remaining mean project duration. For all activities $\left(i_{\eta B}, j_{\eta B}\right)$ with $\rho_{\eta}=1, S_{i_{\eta B}, j_{\eta B}}=t$ is the instant of starting their execution. As for the values 
$p\left(i_{\eta B}, j_{\eta B}\right)$, they are determined by multiple runs of the simulation submodel I with regard for the fixed values of $T\left(i_{\eta B}, j_{\eta B}\right), 1 \leq \xi \leq n_{4}$. The values of $p\left(i_{\eta B}, j_{\eta B}\right)$ are estimated at each instant $t$ of making decision about allocation of the available resources to the queued activities on the basis of the integer programming problem (14)-(16).

\section{Example}

The paper considers a PERT-like network project (Fig. 1) consisting of twenty activities to estimate the efficiency of the developed heuristic algorithm. Its source information is condensed in Table 1. The two emphasized activities consume $A$-resources; the remaining 18 activities consume the $B$-resources of two different types. Therefore, $n=20, n_{A}=2, n_{B}=18, m=2$. The boundary resource values are $R_{1 \text { min }}=30, R_{1 \text { max }}=80, R_{2 \min }=27, R_{2 \max }=80$, the schedule date $D=550$, the confidence probability $p^{* *}$ $=0: 9$, and the fine and project storage estimates on the whole are $C^{*}=1000, C^{* *}=200, C^{* * *}=150$. Values of $r_{i j k}$ are represented in Table 1 except for activities $(4,6)$ and $(7,10)$. The penalty provisions for these activities for idling of the $A$-resources and the cost of lease and maintenance of the $B$-resources are condensed in Table 2 . We note that the parameters of Table 2 were varied in the course of experiment. Sixteen different combinations were included in the experiment (see Table 2). Consideration was given to the uniform distribution over the interval $\left[a_{i j}\right.$; $\left.b_{i j}\right]$ and the normal distribution with the parameters $u_{i j}=$ $0: 5\left(a_{i j}+b_{i j}\right)$ and variance $V_{i j}=\left(b_{i j}-a_{i j}\right)^{2} / 36$ as the distributions of $t_{i j}$. In the problem $P 1$, therefore, the coordinate wise cyclic search of the extremum was carried out in five coordinates[6].

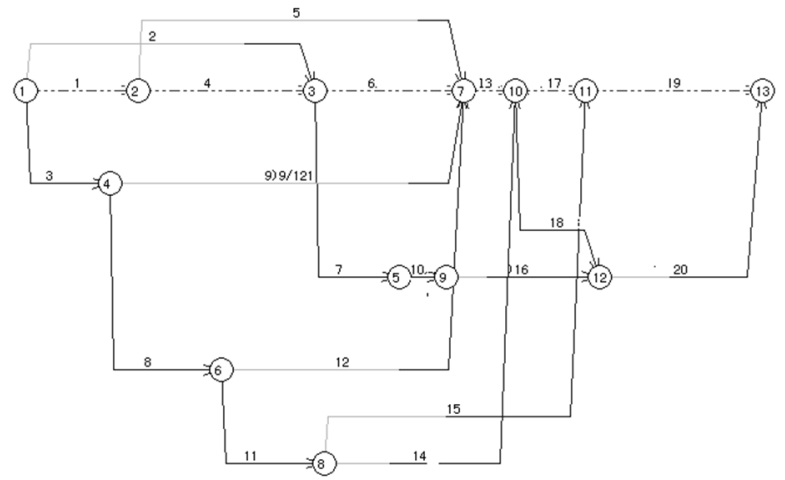

Fig1. PERT-like project network

Table1. Source information about the project

\begin{tabular}{|c|c|c|c|c|}
\hline Activity & $a_{i j}$ & $b_{i j}$ & $r_{1}$ & $r_{2}$ \\
\hline 1 & 24 & 38 & 20 & 10 \\
\hline 2 & 15 & 31 & 17 & 14 \\
\hline 3 & 18 & 30 & 25 & 18 \\
\hline 4 & 38 & 49 & 18 & 20 \\
\hline 5 & 10 & 18 & 23 & 12 \\
\hline 6 & 32 & 49 & 15 & 9 \\
\hline 7 & 18 & 30 & 30 & 22 \\
\hline 8 & 24 & 38 & 0 & 0 \\
\hline 9 & 12 & 26 & 22 & 20 \\
\hline 10 & 10 & 25 & 26 & 17 \\
\hline 11 & 22 & 43 & 30 & 12 \\
\hline 12 & 11 & 34 & 10 & 15 \\
\hline
\end{tabular}

\begin{tabular}{|c|c|c|c|c|}
\hline 13 & 27 & 38 & 0 & 0 \\
\hline 14 & 30 & 48 & 29 & 27 \\
\hline 15 & 24 & 38 & 20 & 10 \\
\hline 16 & 15 & 31 & 17 & 14 \\
\hline 17 & 18 & 30 & 25 & 18 \\
\hline 18 & 38 & 49 & 18 & 20 \\
\hline 19 & 10 & 18 & 23 & 12 \\
\hline 20 & 32 & 49 & 15 & 9 \\
\hline
\end{tabular}

Table2. Varied parameters

\begin{tabular}{|c|c|c|}
\hline Parameters & Values & $\begin{array}{c}\text { Number of } \\
\text { combination }\end{array}$ \\
\hline $\begin{array}{c}A \text {-resources } c\left(i_{\xi A},\right. \\
\left.j_{\xi A}\right) \text { (identical for both } \\
\text { activities })\end{array}$ & $1000 ; 1200$ & 2 \\
\hline Distribution $t_{i j}$ & Uniform; normal & 2 \\
\hline$B$-resources $c_{1}$ & $3 ; 5$ & 2 \\
\hline$B$-resources $c_{2}$ & $3 ; 5$ & 2 \\
\hline
\end{tabular}

At each current point of search $\vec{X}$, four hundred simulation runs were carried out to obtain a representative statistics for estimation of $\bar{C}$. During each run, the optimal submodel at each essential time instant $t$ allocated the $B$ resources on the basis of the problem $P 2$ (14)-(16). The length of the search step $h_{\xi}$ in the problem $P 1$ was accepted to be equal to two for the first iteration and one for all subsequent iterations. The precision of the estimate $\varepsilon>0$ for the general model (1)-(7) was accepted to be equal to $\varepsilon=0.001[7]$.

The following output parameters were established experimentally for each of the 16 combinations:

$\bar{C}$, the minimal mean volume of no operational project costs;

$\bar{p}$, statistical frequency of the probability of timely project execution;

$S$, the instant of project start;

$R_{1}$, the volume of leased $B$-resources of the first kind;

$R_{2}$, the volume of leased $B$-resources of the second kind;

$T(4 ; 6)$, the scheduled delivery time of the $A$-resources for the activity $(4,6)$;

$T(7 ; 10)$, the scheduled delivery time of the $A$ resources for the activity $(7,10)$.

The generalized experimental data are condensed in Table 3 . We note that four iterations were required to obtain the final estimates. In doing so, the results of the fourth and third iterations are virtually identical, and in the course of solving the problem $P 1$ the target function decreased more than two-fold already after the first iteration. In the course of four iterations, the target function decreased by a factor of seven to eight $[8,9]$.

Table3. Output parameters data

\begin{tabular}{|c|c|c|c|}
\hline \multirow{3}{*}{ Distribution } & \multicolumn{3}{|c|}{ Output parameters } \\
\cline { 2 - 4 } & $c\left(i_{\xi_{\xi}}, j_{\xi A}\right)$ & $c_{1}$ & $c_{2}$ \\
\hline \multirow{4}{*}{ uniform } & 1000 & 3 & 5 \\
\cline { 2 - 4 } & 1000 & 5 & 3 \\
\cline { 2 - 4 } & 1200 & 3 & 5 \\
\cline { 2 - 4 } normal & 1200 & 5 & 5 \\
\hline \multirow{4}{*}{} & 1000 & 3 & 3 \\
\cline { 2 - 4 } & 1000 & 5 & 3 \\
\cline { 2 - 4 } & 1200 & 3 & 5 \\
\cline { 2 - 4 } & 1200 & 5 & 5 \\
\hline
\end{tabular}


Table4. Output values data

\begin{tabular}{|c|c|c|c|c|c|c|c|}
\hline \multirow{3}{*}{ Distribution } & \multicolumn{7}{|c|}{ Output values } \\
\hline & \multirow{2}{*}{$S$} & \multirow{2}{*}{$R_{I}$} & \multirow{2}{*}{$R_{2}$} & \multicolumn{2}{|c|}{$T\left(i_{\xi A}, j_{\xi A}\right)$} & \multirow{2}{*}{$C$} & \multirow{2}{*}{$\bar{p}$} \\
\hline & & & & $(4,6)$ & $(7,10)$ & & \\
\hline \multirow{4}{*}{ uniform } & 15 & 43 & 31 & 32 & 104 & 188240 & 0.98 \\
\hline & 14 & 51 & 44 & 34 & 109 & 210311 & 1 \\
\hline & 13 & 48 & 42 & 27 & 98 & 201325 & 0.99 \\
\hline & 13 & 46 & 40 & 30 & 100 & 192220 & 0.98 \\
\hline \multirow{4}{*}{ normal } & 18 & 52 & 44 & 32 & 107 & 150121 & 1 \\
\hline & 16 & 48 & 50 & 31 & 104 & 149211 & 1 \\
\hline & 17 & 47 & 43 & 29 & 102 & 151652 & 1 \\
\hline & 15 & 43 & 44 & 32 & 105 & 156845 & 0.97 \\
\hline
\end{tabular}

\section{Conclusion}

The developed model can be used for planning and supervising one or more Random PERT-like network projects. The model enables one to control several kinds of resources including rare and expensive ones transported from outside for a short time.

For any combination, the mean experimental estimate of the probability of timely project execution exceeds the predefined limit probability that was included as a parameter of the initial problem. Therefore, the constructed model performs probability supervision according to constraint (2).

The normal distribution results in an appreciable (more than by $20 \%$ ) reduction in the no operational costs as compared with the uniform one. Therefore, the normal distribution makes project realization less costly. Therefore, development of more generalized resource models for network projects seems quite topical both from the scientific and applied standpoints.

\section{Acknowledgment}

Thanks to the Architecture Engineering Research Institute of General Logistics Department that give us a lot of help and has prepared and established three books within more than one year. It has taken efforts to bring it to the level of quality as it is today. In this respect, our greatest thank goes not only to all who have contributed and still participate but additionally to all students who give their confidence in our aim to transfer knowledge on a high level.

\section{References}

1. Shtub, A., Bard, J., and Globerson, S., Project Management: Engineering, Technology, and Implementation, New York: Prentice-Hall, 1994, pp. 302-310.

2. Zhan, J., Heuristics for Scheduling ResourceConstrained Projects in MPM Networks, Eur. J. Oper. Res., 1994, vol. 76 (1), pp. 192-205.

3. Golenko-Ginzburg, D. and Gonik, A., Stochastic Network Project Scheduling with Nonconsumable Limited Resources, Int. J. Prod. Econ., 1997, vol. 48, pp. 29-37.

4. Gonik, A., Resource Scheduling Model with Cost Objectives for Stochastic Network Projects, Commun. Dependability Quality Manag., 1999, vol. 2, no. 1, pp. 102-108.

5. Lee, Y., Liang, B., Wang, F., "Some Complexity Metrics for Object-Oriented Programs Based on Information Flow", Proceedings: CompEuro, March, 1993, pp. 302-310.

6. Lorenz, Mark and Kidd, Jeff, Object-Oriented Software Metrics, Prentice Hall Publishing, 1994, pp. 203-210.

7. Tegarden, D., Sheetz, S., Monarchi, D., "Effectiveness of Traditional Software Metrics for Object-Oriented Systems", Proceedings: 25th Hawaii International Conference on System Sciences, January, 1992, pp. 359-368.

8. Williams, John D., "Metrics for Object-Oriented Projects", Proceedings: ObjectExpoEuro Conference, July, 1993, pp. 13-18.

9. Zhuoqun Xia, Zhigang Chen, Xiaoheng Deng. An Enforceable Incentive Scheme in Wireless Multi-path Inter-session Network Coding Game. Journal of Networks, ISSN 1796-2056. Volume: 7, Issue: 2, 2012, pp. 351-355. 